\title{
Negative genetic correlation between traits of the Drosophila head, and interspecific divergence in head shape
}

\author{
FABIAN M. NORRY*, JUAN C. VILARDI \& ESTEBAN HASSON \\ Departamento de Ciencias Biológicas, Facultad de Ciencias Exactas y Naturales, Universidad de Buenos Aires, \\ 1428 Buenos Aires, Argentina
}

\begin{abstract}
For morphological traits that are negatively correlated, the genetic correlation $\left(r_{\mathrm{g}}\right)$ between them might strongly influence patterns of morphological divergence and shape. Here, the pattern of divergence between two sibling species of cactophilic Drosophila, D. buzzatii and D. koepferae, is examined for two traits that are known to be negatively correlated in other Drosophila species: face width $(\mathrm{FW})$ and width of both eyes (EW). Head width (HW, the sum of FW and EW, i.e. the total width of the head capsule) was also examined. Genetic and phenotypic correlations were estimated in the laboratory G2 generation of a sample of wild D. buzzatii derived from a population where D. koepferae is not present. Phenotypic correlations were also estimated in D. buzzatii and D. koepferae from another, very different, population where the species are sympatric. Consistent with studies in other Drosophila species, $r_{\mathrm{g}}$ was negative and significant for the correlation between FW and EW, and positive (but nonsignificant at a matrix-wide $P$-value of 0.05 ) for the correlations of HW with both FW and EW. This well-defined correlation pattern was also consistent with the phenotypic correlations in both D. buzzatii and D. koepferae. No significant difference in these traits was detected between D. buzzatii populations, but head shape has diverged between D. buzzatii and D. koepferae. Specifically, the two negatively correlated traits, FW and EW, have evolved in opposite directions in these two species, with HW showing no significant interspecific difference. The overall picture of this divergence pattern shows a striking concordance with the present evidence of negative correlations between FW and EW, and is consistent with the notion of $r_{\mathrm{g}}$-related constraints on the pattern of interspecific differentiation.
\end{abstract}

Keywords: developmentally related traits, Drosophila head, interspecific divergence, morphology, negative genetic correlation, phenotypic correlation.

\section{Introduction}

Genetic correlations $\left(r_{\mathrm{g}}\right)$ between traits, which arise from pleiotropy or linkage relations among genes controlling the traits, are often considered as factors that affect directions and rates of short-term phenotypic evolution. The short-term response to selection depends not only on the heritabilities of the selected traits but also on the genetic and phenotypic covariances among traits (Falconer, 1989; Roff, 1997; Lynch \& Walsh, 1998). In addition, $r_{\mathrm{g}}$ between pleiotropically related traits can be interpreted as a constraint on their joint microevolution (e.g. Cheverud, 1984; Arnold, 1992). Negative correlations between traits may be of considerable importance in morphological evolution, because negatively correlated

*Correspondence. E-mail: norry@biology.au.dk traits are at least liable to evolve in opposite directions, resulting in a substantial change in shape.

Genetic correlations between morphological traits are more often positive than correlations between other traits (Roff, 1996, 1997), but not all morphological traits are positively correlated. For instance, in developmentally related traits, negative $r_{\mathrm{g}}$ values are expected when the morphological traits arise through a subdivision of a developmental precursor into variable parts (Riska, 1986). Examples of this might be expected in the development in Drosophila, because the imaginal discs, which give rise to the organs and external morphology of the adult fly after metamorphosis, are subdivided into compartments (García-Bellido et al., 1973; Crick \& Lawrence, 1975).

The head capsule is a body part that has received little attention in quantitative studies with Drosophila 


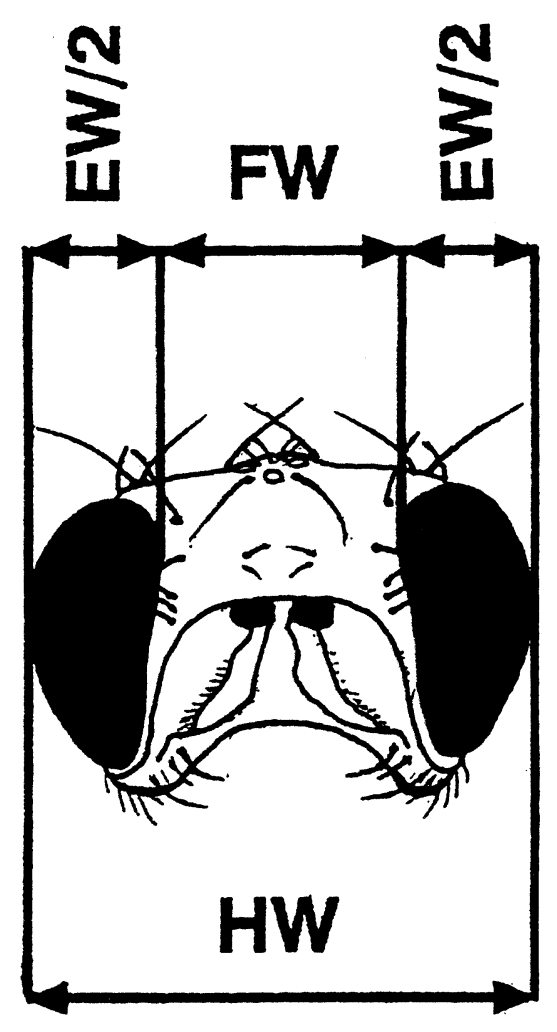

Fig. 1 Description of each measured trait. FW, face width; EW, eye width; HW, head width.

(but see Templeton, 1977; Vall, 1977; Cowley \& Atchley, 1988), at least when compared with the growing research on the quantitative genetics of the wings. This body part is largely produced by a single pair of imaginal discs (the eyeantennal discs, Morata \& Lawrence, 1979), and two traits derived from this developmental precursor, face width and eye width (Fig. 1), are negatively genetically correlated in D. melanogaster (see Cowley's results discussed in Riska, 1986; p. 1307; Cowley \& Atchley, 1990).

Here, the cactophilic fly D. buzzatii, and its sibling species, D. koepferae, are examined for these developmentally related traits. Several aims are addressed. First, we estimate heritabilities and genetic correlations among face width, eye width and head width (Fig. 1) in the laboratory $\mathrm{G} 2$ generation of a sample of wild $D$. buzzatii. Face width is of special interest in this species because males with wider faces have a mating advantage (Norry et al., 1995; Norry \& Vilardi, 1996). Secondly, we estimated phenotypic correlations in both $D$. buzzatii and $D$. koepferae, addressing the hypothesis that phenotypic correlations among these traits are similar, in sign, to their genetic counterparts. Finally, we examined whether the two negatively correlated traits, face width and eye width, have evolved in opposite directions in these two sibling species.

\section{Materials and methods}

\section{Experimental design to estimate genetic parameters}

Genetic parameters were estimated in laboratory-reared flies of D. buzzatii derived from an Argentinian population breeding on Opuntia vulgaris at Arroyo Escobar $\left(34^{\circ} 4^{\prime} \mathrm{S}, 58^{\circ} 7^{\prime} \mathrm{W}\right)$, Province of Buenos Aires, where D. koepferae is absent.

Wild females collected over banana baits (midMarch 1993) were individually kept in $95 \times 20-\mathrm{mm}$ shell vials containing $5 \mathrm{~mL}$ of David's (1962) medium (hereafter referred to as standard vials), obtaining 70 isofemale lines. Seven mating groups were established. Each group was obtained by releasing one male and one virgin female from the $\mathrm{G} 1$ of each isofemale line (140 flies) into a plastic chamber containing two 75-mm-diameter Petri dishes with an egg-laying medium (15 g agar, $75 \mathrm{~mL} \mathrm{95 \%} \mathrm{ethanol,} 15 \mathrm{~mL}$ glacial acetic acid in $1500 \mathrm{~mL}$ water). Food was available in small containers. After $48-72 \mathrm{~h}$, samples of 30 eggs were collected from each chamber and transferred to standard vials. Two sets of these vials were obtained for each mating group. Virgin flies eclosing from these cultures were paired at random by using a balanced $14 \times 14$ factorial design (196 pairs). Each resulting pair was kept in a vial with fresh food for $48 \mathrm{~h}$ prior to transferring both individuals to a $20 \times 30-\mathrm{mm}$ vial containing egg-laying medium. After $36-48 \mathrm{~h}$ both adults were removed and preserved at $-20^{\circ} \mathrm{C}$ for measurement, and after $72-96 \mathrm{~h}$, a sample of 30 first instar larvae was collected from vials containing sufficient larvae (114 out of 196 vials) and transferred to a standard vial. All eclosing offspring were collected (9 vials were discarded due to problems in the cultures), and the parental pairs and four offspring (two flies of each sex) were measured. A total of 105 families that were reared under standardized conditions of larval density were thus obtained. Throughout the experiment, cultures were reared at $24 \pm 1{ }^{\circ} \mathrm{C}$.

The above procedure resulted in an effective random mating (the Pearson correlation between sires and dams was positive but nonsignificant for the traits described in Fig. 1, with the highest $r$-value for EW: $r=0.15$; d.f. $=103$ ). All measurements were performed with a binocular microscope fitted with an ocular micrometer. To measure each trait, the head was removed and observed from the front (Fig. 1) at 100× magnification $(1 \mathrm{~mm}=80$ ocular units). Eye width (EW, width of both eyes) was considered as the sum of both measures, that of the left eye and that of the right eye. Previous studies showed that another related trait, eye length, is positively correlated with both FW and EW (Cowley \& 
Atchley, 1990; Norry et al., 1997). Because we are concerned not with positive correlations but with negatively correlated traits, data on this trait were omitted in the present study.

\section{Collection of strains derived from the Quilmes population}

The same traits were also scored in males of $D$. buzzatii and $D$. koepferae derived from the Quilmes population $\left(26^{\circ} 6^{\prime} \mathrm{S}, 65^{\circ} 9^{\prime} \mathrm{W}\right)$, where both sibling species are sympatric. This population and that of Arroyo Escobar (see above) are approximately $1150 \mathrm{~km}$ apart (see Hasson et al., 1995; for information about the many differences between these populations). Previous work on this population showed that the pattern of morphometric differences between these species is equal in both sexes (Norry, 1995), particularly for the head traits examined here. The flies examined were the offspring of a random mating performed with the laboratory G2 generation of 73 (D. koepferae) or 48 (D. buzzatii) isofemale lines from wild inseminated females collected over banana bait buckets (species were identified by examining the male genitalia, see Vilela, 1983). The random mating was performed within each species by releasing three virgin flies of each sex and isofemale line into an egg-collecting chamber $(100 \times 200 \times 300 \mathrm{~mm})$. Samples of 30-40 firstinstar larvae were collected daily from this chamber and transferred to each of 10 standard vials for optimal growth at $24 \pm 1{ }^{\circ} \mathrm{C}$. For each species, 50 randomly chosen males ( 5 from each vial) emerging from these cultures (i.e. the laboratory G3 generation) were measured.

\section{Estimation of genetic parameters and phenotypic correlations}

Genetic parameters and their standard errors were estimated using the standard parametric procedures described in Falconer (1989). All data (in ocular units) were $\log _{\mathrm{e}}$-transformed and corrected to remove sexual dimorphism in the mean value of each trait. This correction was done by adding to male measurements the difference between female and male means.

Heritabilities were estimated by regressing mean values of offspring on mid-parent values (Falconer, 1989). This method assumes that the sexes do not significantly differ in phenotypic variance (Falconer, 1989), which was verified (Bartlett's test results for $\log _{\mathrm{e}}$-transformed data on the 630 individuals measured in this study are: $\chi^{2}=3.32$ for $\mathrm{FW}, \chi^{2}=3.11$ for $\mathrm{EW} ; \chi^{2}=0.90$ for $\mathrm{HW}$. In all cases, d.f. $=1 ; P>0.05$ ). In addition, we have no evidence of additive X-linked variance in these traits in the same population (Norry et al., 1997; present study).

Genetic correlations $\left(r_{\mathrm{g}}\right)$ were computed from midparent-offspring covariances (Falconer, 1989), obtaining two estimates for each pair of traits $\mathrm{X}$ and Y:

$r_{1}=\operatorname{cov}\left(\mathrm{X}^{\prime \prime}, \mathrm{Y}^{\prime}\right) /\left[\operatorname{cov}\left(\mathrm{X}^{\prime \prime}, \mathrm{X}^{\prime}\right) \operatorname{cov}\left(\mathrm{Y}^{\prime \prime}, \mathrm{Y}^{\prime}\right)\right]^{1 / 2}$

$r_{2}=\operatorname{cov}\left(\mathrm{X}^{\prime}, \mathrm{Y}^{\prime \prime}\right) /\left[\operatorname{cov}\left(\mathrm{X}^{\prime \prime}, \mathrm{X}^{\prime}\right) \operatorname{cov}\left(\mathrm{Y}^{\prime \prime}, \mathrm{Y}^{\prime}\right)\right]^{1 / 2}$,

where " refers to mid-parent and ' to offspring values. The average of $r_{1}$ and $r_{2}$ is the estimated genetic correlation. Standard errors of $r_{\mathrm{g}}$ were estimated using Reeve's (1955) formula:

$\mathrm{SE}\left(r_{\mathrm{g}}\right)=\left[\left(1 \quad r_{\mathrm{g}}^{2}\right) / \sqrt{2}\right]\left[\operatorname{SE}\left(h_{x}^{2}\right) \operatorname{SE}\left(h_{y}^{2}\right) /\left(h_{x}^{2} h_{y}^{2}\right)\right]^{1 / 2}$.

Phenotypic correlations were computed as the Pearson product-moment correlation between traits. Statistical significance of both genetic and phenotypic correlations was assessed for a matrix-wide $P$-value of 0.05 , using the sequential Bonferroni test (Rice, 1989).

Precise estimation of genetic correlations between the sexes requires a half-sib design (e.g. Cowley \& Atchley, 1988). Therefore, no such estimate was obtained.

\section{Morphological divergence}

Interspecific differences in each trait were estimated in males, using:

$\mathrm{DZ}=\mathrm{X}_{i} \quad \mathrm{X}_{j}$,

where $\mathrm{X}_{i}$ and $\mathrm{X}_{j}$ are, respectively, the mean values (in $\mathrm{mm}$ ) in D. buzzatii and D. koepferae. In this study, the analysis is arbitrary with respect to whether D. koepferae or D. buzzatii is the 'ancestral' species. In particular, we are concerned not with a retrospective selection analysis (Lande, 1979) but with the hypothesis that negatively correlated traits have evolved in opposite directions, which may be verified by examining whether the traits differ, in sign, for the DZ-values.

One-way ANOva and Scheffe multiple comparisons for heterogeneity of means were performed for each trait. As the sample size of males of each species was 50 for the Quilmes population (see above), the analysis was balanced by taking a randomly chosen sample of 50 male parents of the heritability experiment for the Arroyo Escobar population. 


\section{Results}

\section{Genetic and phenotypic correlations between the traits}

Heritability estimates were all significant, with moderate to low values for all traits (Table 1). The slopes of damoffspring regressions were roughly similar to the slopes of sire-offspring regressions (results not shown), with no significant difference in an analysis of covariance of the slopes (the highest $F$-value was obtained for EW: $F_{1,206}=2.50, P>0.05$ ). Thus, there is no apparent evidence of additive X-linked variance (see Norry et al., 1997; for similar results in other traits).

Genetic correlations were moderate in magnitude, with only one of the $r_{\mathrm{g}}$-values being negative and significant at a matrix-wide $P$-value of 0.05 (Table 1 ). This negative $r_{\mathrm{g}}$ corresponds to the correlation between FW and EW (Table 1).

A phenotypic correlation pattern is evident in D. buzzatii from Arroyo Escobar, again with a negative value for the correlation between FW and EW, and positive values for the correlations of $\mathrm{HW}$ with both $\mathrm{FW}$ and EW (Table 1). This phenotypic correlation pattern is also observed in laboratory-reared males of both D. koepferae and D. buzzatii derived from the Quilmes population (Table 2).

\section{Morphological divergence}

Means and SD of the studied traits are shown in Table 3 for males, in both $D$. buzzatii and D. koepferae. Differences in trait means are significant between these

Table 1 Heritabilities (on diagonal), and genetic (below diagonal) and phenotypic (above diagonal) correlations among three head traits in laboratory-reared $D$. buzzatii derived from the Arroyo Escobar population. Phenotypic correlations are for parents and offspring combined (630 flies of the heritability experiment). Standard errors are in italics

\begin{tabular}{llcc}
\hline & HW & FW & EW \\
\hline HW & $0.30^{* *}$ & $\mathbf{0 . 5 4} 4^{* * *}$ & $\mathbf{0 . 6 2 * * *}$ \\
& 0.09 & & \\
FW & $0.35^{*}$ & $0.26^{* *}$ & $\mathbf{- 0 . 3 1 * *}$ \\
& 0.20 & 0.09 & \\
EW & $0.44^{*}$ & $\mathbf{- 0 . 4 8}^{*}$ & $0.22^{*}$ \\
& 0.21 & 0.21 & 0.10 \\
\hline
\end{tabular}

Boldface correlations are significant at a matrix-wide $P$-value of 0.05 (sequential Bonferroni test). Individual

$P$-values (i.e. without applying a Bonferroni technique) are:

${ }^{*} P<0.05 ;{ }^{* *} P<0.005 ; * * * P<0.0005$. species, except for HW (Table 3). Because no significant difference was detected between D. buzzatii populations (Table 3), DZ-values are shown only for the betweenspecies comparisons (Fig. 2). A divergence pattern is evident: FW has evolved in an opposite direction to EW (Fig. 2), with these traits exhibiting highly significant differences between species (Table 3). The apparent stability in HW is largely a by-product of this pattern of opposite changes in $\mathrm{FW}$ and $\mathrm{EW}$, as $\mathrm{DZ}_{\mathrm{HW}^{-} \text {-values }}$ are approximately equivalent to the sum of the respective $\mathrm{DZ}_{\mathrm{FW}}$ and $\mathrm{DZ}_{\mathrm{EW}}$-values (Fig. 2).

\section{Discussion}

A negative genetic correlation was found between face width and eye width in laboratory-reared flies of D. buzzatii derived from the Arroyo Escobar population. Phenotypic correlations between these traits were also negative in laboratory-reared flies of $D$. buzzatii

Table 2 Phenotypic correlations among three head traits in laboratory-reared males of $D$. buzzatii (below diagonal) and $D$. koepferae (above diagonal) derived from the Quilmes population

\begin{tabular}{lccc}
\hline & HW & FW & EW \\
\hline HW & & $\mathbf{0 . 5 7 * * *}$ & $\mathbf{0 . 7 4 * * *}$ \\
FW & $\mathbf{0 . 5 4} * * *$ & & $-\mathbf{0 . 3 1} * *$ \\
EW & $\mathbf{0 . 7 1} * * *$ & $-0.20^{*}$ & \\
\hline
\end{tabular}

Boldface values are significant at a matrix-wide $P$-value of 0.05 (sequential Bonferroni test). Individual $P$-values (i.e. without applying a Bonferroni technique) are: $* P<0.05 ; * * P<0.005 ; * * * P<0.0005$.

Table 3 Mean value (in mm) and SD (italics) for three developmentally related traits of the head capsule in laboratory-reared males of: D. koepferae from the Quilmes population (DKQ), D. buzzatii from the Quilmes population (DBQ), and D. buzzatii from the Arroyo Escobar population (DBAE). Results of ANOvA and Scheffe multiple comparisons for heterogeneity of means are also shown. Homogeneous means are connected by a continuous underline

\begin{tabular}{lcccc}
\hline Trait & DKQ & DBQ & DBAE & $\mathrm{F}_{2 ; 147}$ \\
\hline HW & 0.939 & 0.940 & 0.940 & 0.16 \\
& 0.027 & 0.020 & 0.026 & \\
FW & 0.420 & $\underline{0.408}$ & 0.408 & $5.46^{*}$ \\
& 0.018 & 0.015 & 0.018 & \\
EW & 0.516 & $\underline{0.532}$ & 0.530 & $8.57^{* *}$ \\
& 0.019 & 0.015 & 0.016 & \\
\hline
\end{tabular}

${ }^{*} P=0.005 ; * * P=0.0004$.

(c) The Genetical Society of Great Britain, Heredity, 85, 177-183. 
Fig. 2 Difference (DZ, in $\mathrm{mm}$ ) in each trait between laboratory-reared males of D. buzzatii and D. koepferae derived from two natural populations: DBAE, D. buzzatii from the Arroyo Escobar population; DBQ, D. buzzatii from the Quilmes population; DKQ, D. koepferae from the Quilmes population. Abbreviations of traits are given in Fig. 1.

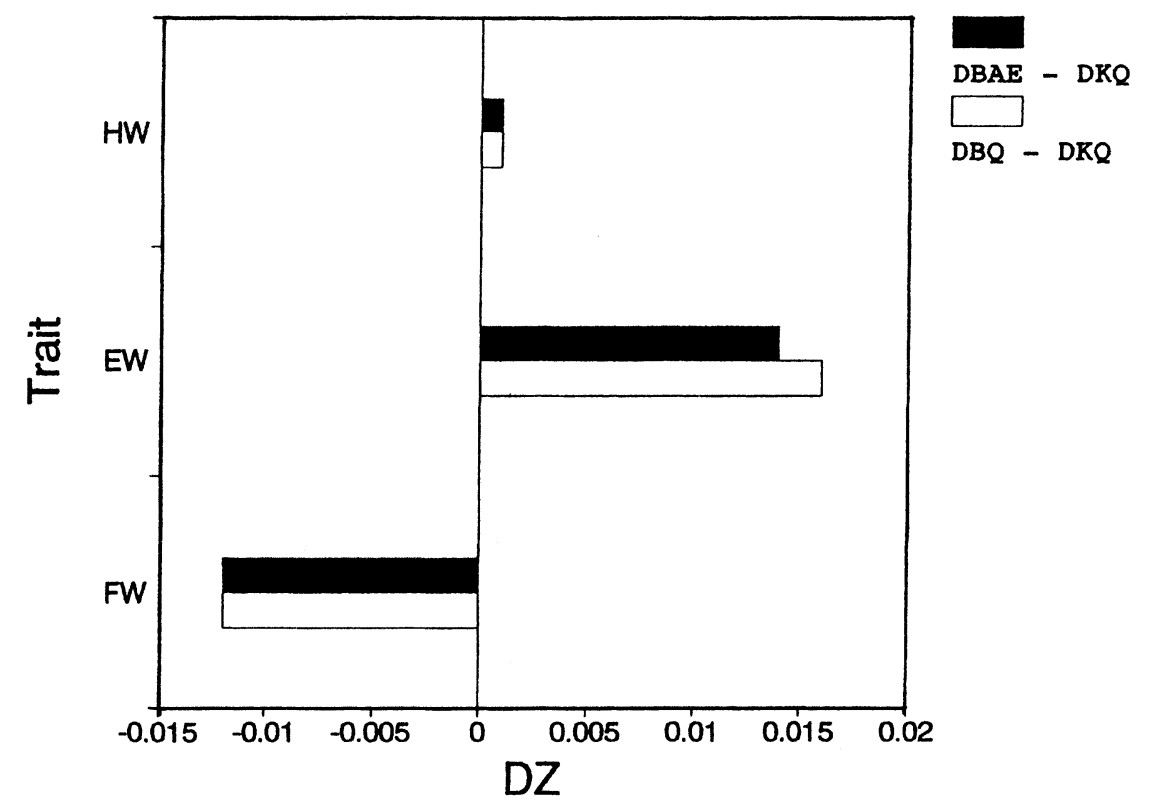

and D. koepferae derived from a different population (Tables 1 and 2). Similar results were obtained by David Cowley (discussed in Riska, 1986; p. 1307; see also Cowley \& Atchley, 1990) in D. melanogaster. Thus, negative correlations between face width and eye width may be widespread in Drosophila.

This negative correlation is even more apparent in the pattern of morphological divergence between species. Head shape has diverged between D. buzzatii and D. koepferae. Specifically, FW and EW exhibit negatively correlated and highly significant DZ-values (Fig. 2), with HW showing no significant difference between populations/species. Thus, the two negatively correlated traits, FW and EW, have evolved in opposite directions in these two sibling species. The very consistent pattern of interspecific differentiation (Fig. 2) is supporting evidence of constraints on the range of likely combinations of mean traits. The overall picture of this divergence pattern is consistent with the present evidence for negative correlations between FW and EW. In addition, an apparent way for $r_{\mathrm{g}}$-related constraints to emerge when, as is suggested for $\mathrm{HW}$ (Fig. 2), the apparently more constrained trait is the result (e.g. the sum) of two negatively correlated traits.

Because $r_{\mathrm{g}} \mathrm{s}$ were estimated in $D$. buzzatii but not in D. koepferae, a possible difficulty in these comparisons is that genetic correlations among the traits could differ, in sign, between these sibling species. However, this possibility is unlikely, because the genetic correlation between face width and eye width is negative also in the less closely related D. melanogaster (Cowley \& Atchley, 1990). Furthermore, it is remarkable that for our set of studied traits, the genetic correlations estimated in
D. buzzatii are similar, in sign, to their phenotypic counterparts, not only in D. buzzatii but also in both its sibling species D. koepferae (Tables 1 and 2) and the less closely related D. melanogaster (Cowley \& Atchley, 1990). Thus, it is unlikely that the overall picture of negative correlations suggested by the results would be incorrect for our two sibling species. In addition, the negatively correlated traits, FW and EW, have evolved in opposite directions in many Drosophila species, including $D$. melanogaster and its sibling species D. simulans (results not shown).

Traits representative of overall size of head, thorax and wings tend to be positively correlated in Drosophila, at least in D. melanogaster (Cowley \& Atchley, 1990) and D. buzzatii (Norry et al., 1997). In addition, while functional (selective) relationships between traits may have substantial effects on $r_{\mathrm{g}}$ values (Sheridan \& Barker, 1974), development is probably also a major determinant of pleiotropic relationships between morphological traits in Drosophila (Cowley \& Atchley, 1990). The traits examined in the present study constitute an example of developmentally related traits, as these traits are derived from a common precursor, the eye-antennal discs (Morata \& Lawrence, 1979). It is possible that negative correlations between face width and eye width reflect subdivision of the imaginal disc cells into a population that forms the multifaceted eye and a population that forms tissue that is not destined to form eye, as first suggested by Cowley \& Atchley (1990). In fact, negative $r_{\mathrm{g}}$ values are predicted when the traits arise through a subdivision of a developmental precursor into variable parts (Riska, 1986), and negative correlations between face and eye widths 
are probably widespread in Drosophila (Cowley \& Atchley, 1990; present study). Alternatively, negative correlations may also be the result of competition between processes for a resource (Atchley, 1987) or functional relationships between the traits (but see Sheridan \& Barker, 1974; for expected $r_{\mathrm{g}}$ values under two-trait selection with a resource competition model). The well-known case of $D$. heteroneura and $D$. silvestris, with the putative importance of behaviour in the topography of eyes for mate recognition (Templeton, 1977; but see Price \& Wake, 1995), might suggest that some functional relationships are possible between the studied traits. While these traits are correlated with mating success in D. buzzatii (Norry et al., 1995; 1996), they could be influenced either by developmental or functional effects, or both. Another interesting example of negative $r_{\mathrm{g}}$ that probably reflects developmental effects is the correlation between the proximal wing length and the distal wing length in D. buzzatii (Thomas \& Barker, 1993; Loeschcke et al., 1999).

Further observations are needed to test these alternative explanations. However, despite some uncertainty over the correlation's cause(s), our present observations do indicate that the two negatively correlated parts of the Drosophila head evolve typically in opposite directions, resulting in substantial interspecific divergence in head shape. For morphometric traits that are consistently negatively correlated as in the present study, their resulting patterns of interspecific divergence and shape variation could severely be biased in a trend that is consistent with the negative sign of $r_{\mathrm{g}}$.

\section{Acknowledgements}

We thank Juan J. Fanara for help in rearing flies of the Quilmes population, Dr David Cowley for comments on the results, and Professor J.S.F. Barker for helpful comments on the manuscript. This research was supported by Universidad de Buenos Aires grants EX179/ 95 to J.C.V. and EX099 to E.H.

\section{References}

ARNOLD, S. J. 1992. Constraints on phenotypic evolution. Am. Nat., 140 (Suppl.), s85-s87.

ATCHLEY, W. R. 1987. Developmental quantitative genetics and the evolution of ontogenics. Evolution, 41, 316-330.

CHEVERUD, J. M. 1984. Quantitative genetics and developmental constraints on evolution by selection. J. Theor. Biol., 110, $155-171$.

COWLEY, D. E. AND ATCHLEY, W. R. 1988. Quantitative genetics of Drosophila melanogaster. II. Heritabilities and genetics correlations between sexes for head and thorax traits. Genetics, 119, 421-433.
COWLeY, D. E. AND ATChley, w. R. 1990. Development and quantitative genetics of correlation structure among body parts of Drosophila melanogaster. Am. Nat., 135, 242268.

CRICK, F. H. C. AND LAWRENCE, P. A. 1975. Compartments and polyclones in insect development. Science, 189, 340-347.

DAVID, J. 1962. A new medium for rearing Drosophila in axenic conditions. Dros. Inf. Serv., 36, 128.

FALCONER, D. S. 1989. Introduction to Quantitative Genetics, 3rd Edn. John Wiley, New York.

GARCíA-BEllido, A., RIPOLl, P. AND MORATA, G. 1973. Developmental compartmentalisation of the wing disc of Drosophila. Nature (New Biology), 245, 251-253.

HASSON, E., RODRIGUEZ C., FANARA, J. J., NAVEIRA, H. AND FONTDEVILA, A. 1995. The evolutionary history of Drosophila buzzatii. XXVI. Macrogeographic patterns of inversion polymorphism in New World populations. J. Evol. Biol., 8, 369-384.

LANDE, R. 1979. Quantitative genetic analysis of multivariate evolution, applied to brain: body size allometry. Evolution, 33, 402-416.

LOESCHCKE, V., BUNDGAARD, J. AND BARKER, J. S. F. 1999. Reaction norms across, and genetic parameters at different temperatures for, thorax and wing size traits in Drosophila aldrichi and D. buzzatii. J. Evol. Biol., 12, 605-623.

LYNCH, M. AND WALSH, B. 1998. Genetics and Analysis of Quantitative Traits. Sinauer Associates, Sunderland, MA.

MORATA, G. AND LAWRENCE, P. A. 1979. Development of the eye-antennal imaginal disc of Drosophila. Dev. Biol., 70, 355-371.

NORRY, F. M. 1995. Selección sexual relacionada con el tamaño corporal, parámetros genético-cuantitativos multivariados, y divergencia morfométrica multivariada entre poblaciones de dos especies cactófilas del género. Drosophila (Diptera: Drosophilidae). PhD dissertation, Universidad de Buenos Aires.

NORRY, F. M., VILARDI, J. C., FANARA, J. J. AND HASSON, E. 1995. Courtship success and multivariate analysis of sexual selection on morphometric traits in Drosophila buzzatii (Diptera: Drosophilidae). J. Insect Behav., 8, 219-229.

NORRY, F. M. AND VILARDI, J. C. 1996. Size-related sexual selection and yeast diet in Drosophila buzzatii (Diptera, Drosophilidae). J. Insect Behav., 9, 329-338.

NORRY, F. M., VILARDI, J. C. AND HASSON, E. 1997. Genetic and phenotypic correlations among size-related traits, and heritability variation between body parts in Drosophila buzzatii. Genetica, 101, 131-139.

PRICE, D. K. AND WAKE, C. R. B. 1995. Behavioral reproductive isolation in Drosophila silvestris, D. heteroneura, and their F1 hybrids (Diptera: Drosophilidae). J. Insect Behav., 8, 595-616.

REEVE, E. C. R. 1955. The variance of the genetic correlation coefficient. Biometrics, 11, 357-374.

RICE, W. R. 1989. Analyzing tables of statistical tests. Evolution, 43, 223-225.

RISKA, B. 1986. Some models for development, growth, and morphometric correlation. Evolution, 40, 1303-1311.

ROFF, D. A. 1996. The evolution of genetic correlations: an analysis of patterns. Evolution, 50, 1392-1403. 
ROFF, D. A. 1997. Evolutionary Quantitative Genetics. Chapman \& Hall, New York.

SHERIDAN, A. K. AND BARKER, J. S. F. 1974. Two-trait selection and genetic correlation. II. Changes in genetic correlations during two-trait selection. Aust. J. Biol. Sci., 27, 89-101.

TEMPLETON, A. R. 1977. Analysis of head shape differences between two interfertile species of Hawaiian Drosophila. Evolution, 31, 630-641.
THOMAS, R. H. AND BARKER, J. S. F. 1993. Quantitative genetic analysis of the body size and shape of Drosophila buzzatii. Theor. Appl. Genet., 85, 598-608.

VALL, F. C. 1977. Genetic analysis of the morphological differences between two interfertile species of Hawaiian Drosophila. Evolution, 31, 611-629.

vilelA, C. R. 1983. A revision of the Drosophila repleta species (Diptera-Drosophilidae). Rev. Brasil. Entomol., 27, 1-114. 